\title{
Abdominal Flank Bulging after Lateral Retroperitoneal Approach: A Case Report
}

\author{
Jeong-Hoon Choi, ${ }^{1}$ Jee-Soo Jang, ${ }^{1}$ and II-Tae Jang ${ }^{2}$
}

\begin{abstract}
The lateral transpsoas approach to access the vertebrae obviates the need for an approach surgeon and minimizes muscular disruption, thus allowing for quicker recovery. Several reports on the lateral transpsoas procedure have described few complications. However, the development of an unsightly and painful abdominal flank bulge is a largely under-recognized and very rare complication of the lateral transpsoas approach. A 59-year-old man suffered from back pain and bilateral posterior leg pain. Computed tomography (CT) scan and MRI showed retrolisthesis at L3-4, L2 wedge vertebrae with kyphosis, left L4 screw loosening, and L3-4 disc herniation with central canal stenosis. L2 corpectomy and L3-4 DLIF and posterior fusion to T12 for kyphosis correction were performed. For the lateral approach, resection of the T11 rib was performed. One month later, he developed left abdominal flank bulging below the lateral approach site, which was aggravated by walking, coughing, defecating, constipation, and eating. CT scan showed left abdominal flank bulging accompanied by abdominal muscle thinning. We believe that this complication is caused by denervation of the abdominal musculature after injury to the T11 intercostal nerves.
\end{abstract}

Keywords: direct lateral interbody fusion; abdominal flank bulging; lateral retroperitoneal approach; postoperative complication; nerve denervation injury

\section{Introduction}

The minimal invasive lateral transpsoas approach, as known as direct lateral interbody fusion (DLIF) or extreme lateral interbody fusion (XLIF), is a relatively new surgical technique that allows access to the lumbar disc space and vertebral body without an extensive muscle dissection. DLIF has become an increasingly popular approach for achieving interbody fusion over the past decade because it can avoid the morbidity of anterior approaches. This procedure has been shown to have decreased blood loss and transfusion rates, lower rates of postoperative back pain, quicker recovery, and shorter hospital stays. ${ }^{1)}$ It uses a lateral retroperitoneal corridor that has less risk of injury to the peritoneal contents and the hypogastric sympathetic plexus as compared to more anterior interbody approaches.

${ }^{1}$ Department of Neurosurgery, Nanoori Hospital, Suwon, Gyunggi province, Korea

${ }^{2}$ Department of Neurosurgery, Nanoori Hospital, Seoul, Korea

Received: March 10, 2016; Accepted: July 6, 2016
However, the DLIF technique can lead to lateral transpsoas approach-related complications with the potential for significant neurologic morbidity. ${ }^{2,3)}$ The most common reported complications of the lateral transpsoas approach are ipsilateral anterior thigh pain and psoas major muscle weakness, both of which are related to splitting the psoas muscle fibers upon approaching the spine and minor trauma to the lumbar plexus. ${ }^{4)}$ An unsightly and painful abdominal flank bulge is a largely under-recognized and very rare complication of the lateral transpsoas approach. Postoperatively, abdominal muscle laxity and flank bulge results in an uncomfortable, asymmetrical cosmetic deformity and patient dissatisfaction with physical appearance. We describe a case of flank bulging after DLIF along with a literature review.

\section{Case Report}

A 59-year-old man presented with a 10-month history of back pain, bilateral buttock pain, and bilateral radiating posterior leg pain and numbness. The patient exhibited a limping gait due to the pain. He had undergone an L5-S1 posterior lumbar interbody fusion surgery 7 years ago at another hospital. He was treated with many different kinds of conservative therapies. However, the pain did not decrease. Magnetic resonance imaging (MRI) showed L4-5 disc herniation with a bilateral foraminal stenosis and an L2 wedge vertebrae with kyphosis (Fig. 1). He underwent anterior lumbar interbody fusion (ALIF) at the L4-5 level with bilateral foraminal decompression and posterior screw fixation (Fig. 2). After the operation, the patient's preoperative symptoms improved.

However, 8 months later, he complained of buttock and posterior leg pain on the left side. Radiography and a computed tomography (CT) scan showed retrolisthesis at the L3-4 level and a position change of the left L4 screw with periscrew halo (Fig. 3). We assumed that L3-4 retrolisthesis with stenosis developed due to the L2 wedge vertebrae with kyphosis. Therefore, we decided to perform an L2 corpectomy and L3-4 DLIF with a lateral transpsoas approach and fusion extension to T12 for kyphosis correction (Fig. 4). The incision was made along the 11th rib and coursed anteriorly and inferiorly around the abdomen, extending medially to the lateral aspect of the rectus sheath. The three layers of the lateral abdominal wall muscle were then split between the muscle fibers to allow entry into the retroperitoneum. We did not use electrocautery. A dissector was used to perform periosteal dissection, stripping the intercostal muscles and intercostal neurovascular bundle off the posterior aspect of the rib. We needed to resect the 11th rib to provide a wide operational view for $\mathrm{L} 2$ 

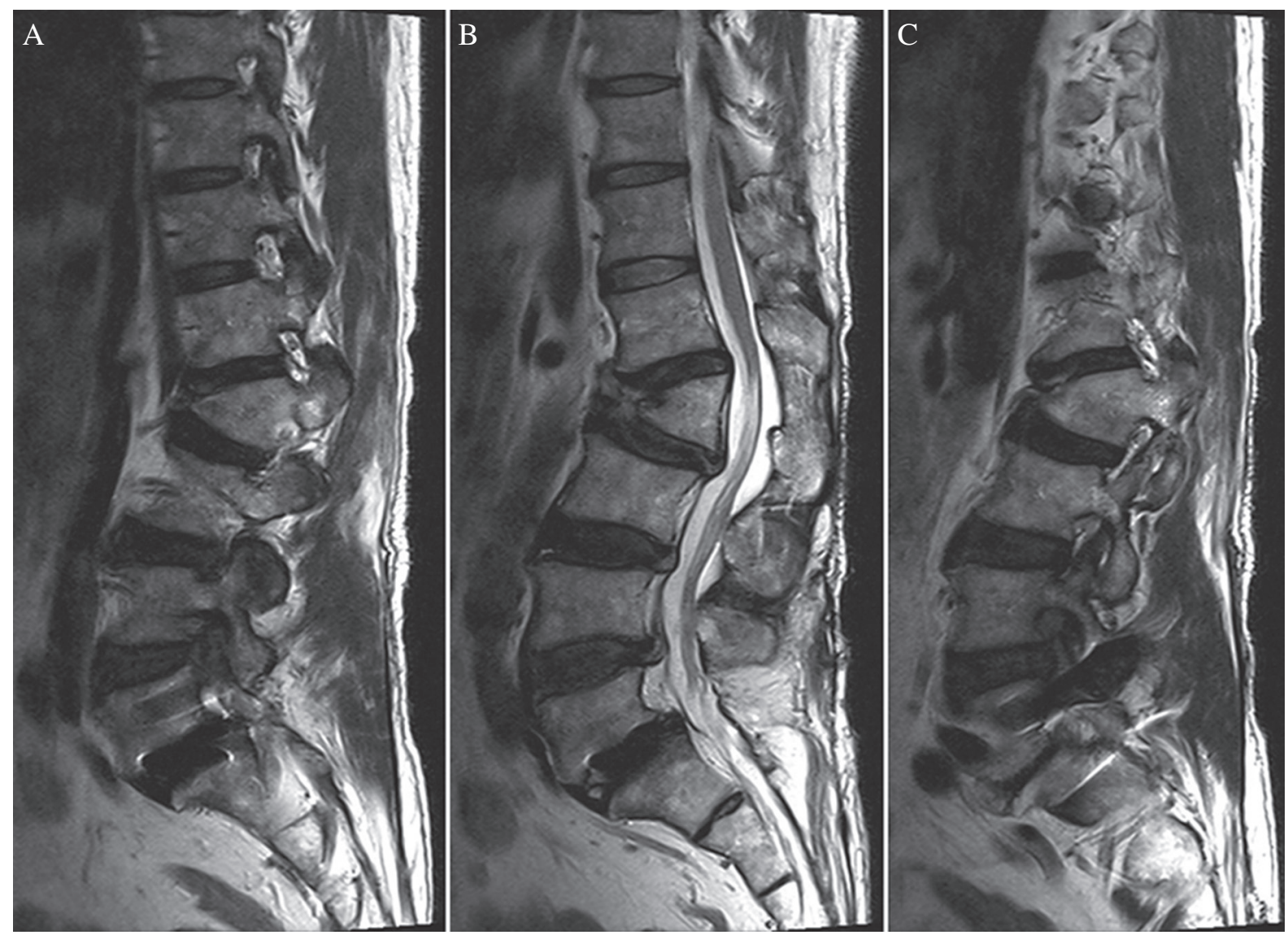

Fig. 1 Sagittal MRI shows L4-5 disc herniation with bilateral foraminal stenosis and L2 wedge vertebrae with kyphosis. A: right foraminal area, B: central, C: left foraminal area.

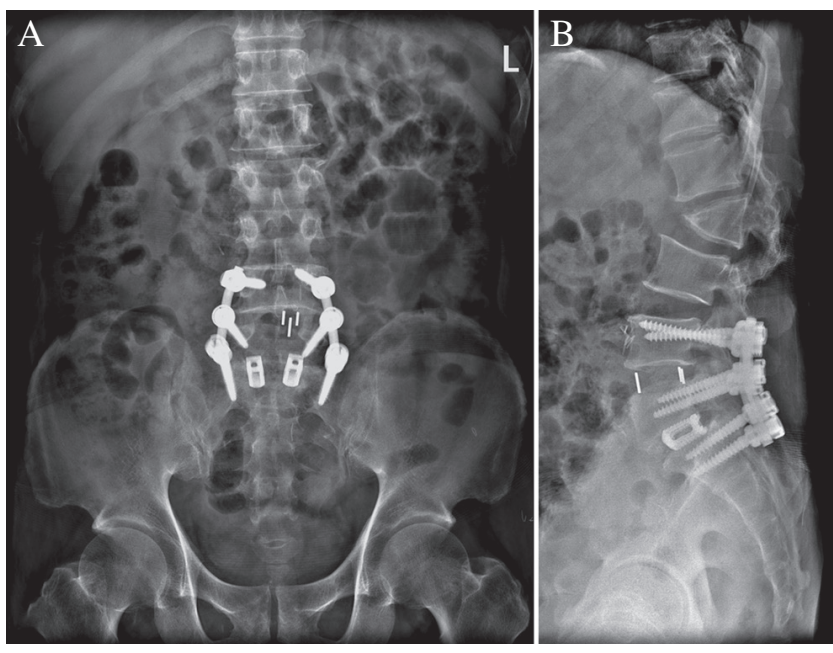

Fig. 2 Postoperative plan radiography shows interbody cage insertion at L4-5 level with fusion extension to L4. A: anterior-posterior view, B: lateral view.

corpectomy and cage insertion. Therefore, we resected the 11 th rib and retracted the 10th and 12 th rib, including the 11th intercostal neurovascular bundle, for a long period in order to perform the L2 corpectomy and cage insertion.

After the operation, the patient's symptoms improved and he was discharged 10 days later. One month postsurgery he experienced left abdominal flank discomfort and bulging below the skin incision on the lateral approach side (Fig. 5). The abdominal flank bulging showed an oblique and dermatomal pattern. It was aggravated by walking, coughing, defecating, constipation, and eating. The patient experienced hypoesthesia around the bulge. A CT scan showed left abdominal flank bulging accompanied by abdominal muscle thinning, with no evidence of an abdominal facial defect or a bowel hernia (Fig. 6). The condition has not changed over 17 months of post-surgical follow-up. We are continuing to observe the patient.

\section{Discussion}

Injury to the nerves of the lumbar plexus traversing the psoas muscle is a well-documented surgical complication. New onset thigh numbness, dysesthesias, pain, and/or weakness are reported by $30.4 \%$ of patients undergoing lateral retroperitoneal approach surgery. ${ }^{5)}$ In addition to these complications, postoperative pain due to injury to the intercostal nerves during thoracic surgery is a well-known complication. The higher incidence of chronic post-thoracotomy pain syndrome suggests surgical nerve damage is common. Retractor-induced direct intercostal nerve damage takes place in all patients and results in indirect nerve damage in $44 \%$ of patients. ${ }^{6}$ Around $50 \%$ of patients undergoing thoracic surgery suffer from various intensities of chronic post-thoracotomy pain syndrome, most likely due to intercostal nerve damage. ${ }^{7)}$ 

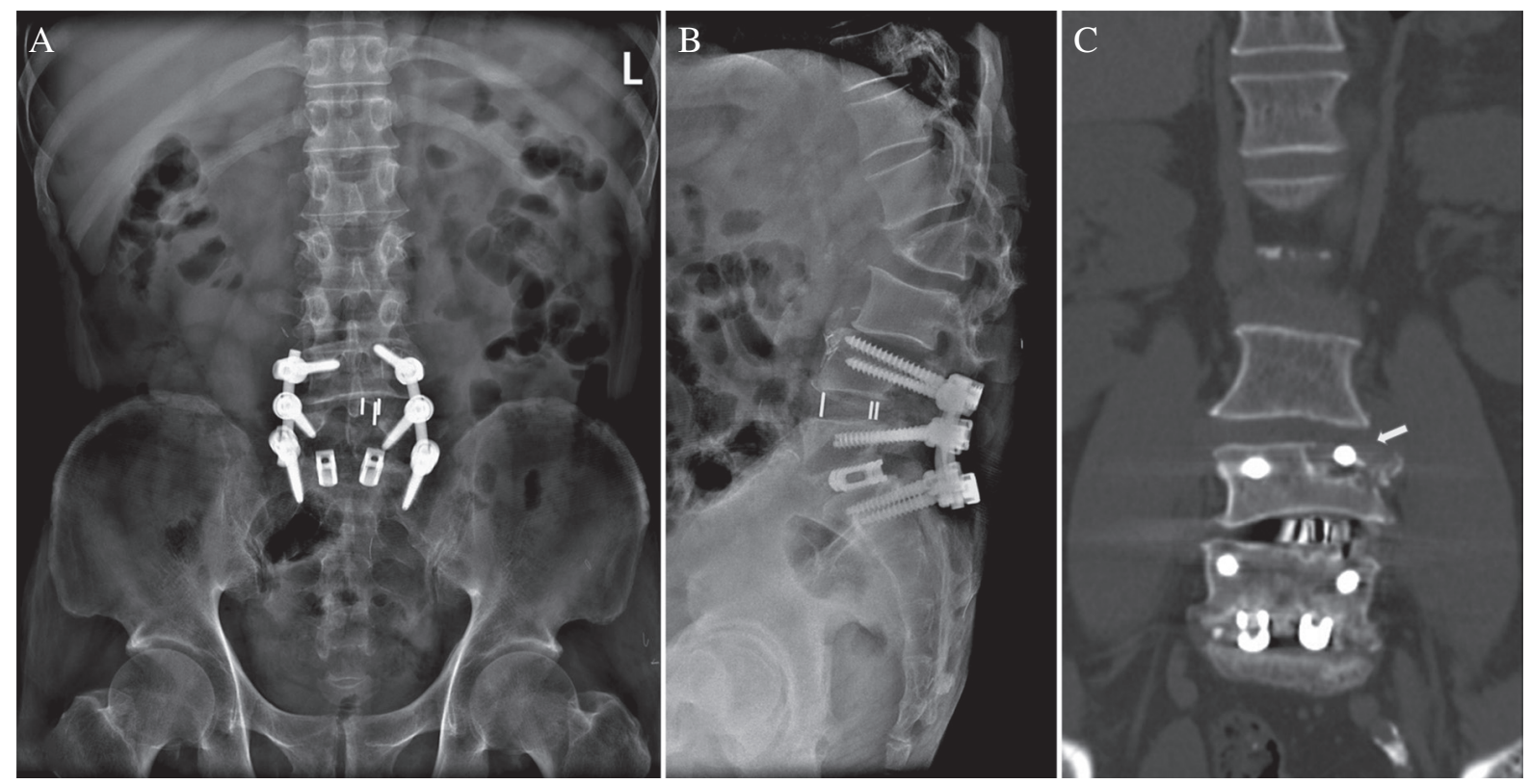

Fig. 3 After 8 months of operation, follow-up plan radiography showed retrolisthesis at L3-4 level and left L4 screw position change. CT scan shows periscrew hallo (white arrow). A: anterior-posterior view, B: lateral view, C: CT scan coronal view.

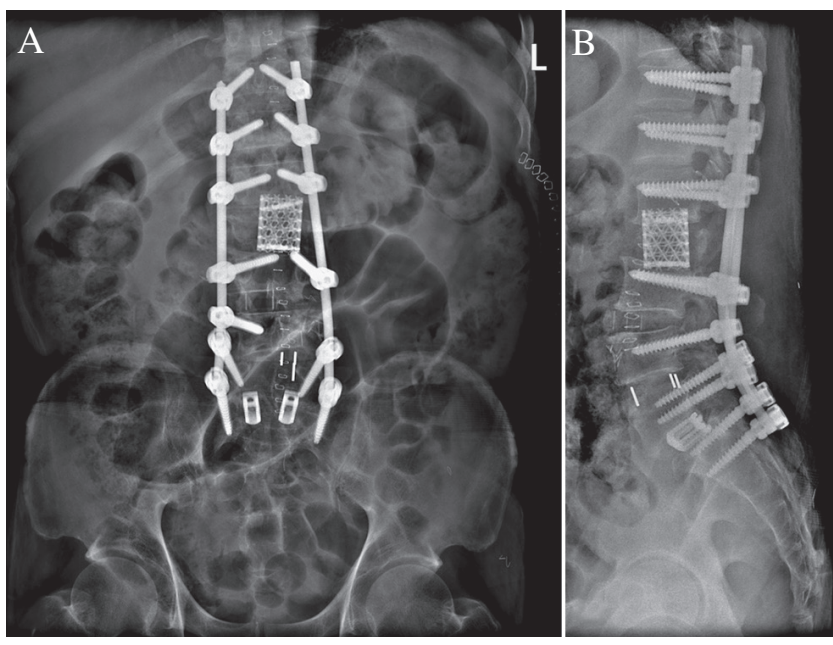

Fig. 4 Plan radiography shows L2 corpectomy and mesh cage insertion, L3-4 interbody cage insertion, fusion extension to T12 level, and kyphosis correction state. A: anterior-posterior view, B: lateral view.

Innervation of the abdominal rectus and internal and external oblique muscles is mostly established by the $\mathrm{T} 7$ to T12 intercostal nerves. These nerves supply the rectus abdominis muscle and internal and external oblique muscles and terminate in the anterior cutaneous branches of the abdomen. Cadaveric dissection studies and intraoperative ElectroMyoGraphy (EMG) evaluations provided detailed knowledge of the anatomy of the anterolateral abdominal wall and its innervation. ${ }^{8)}$ Cadaveric dissections have shown that the most significant intercostal nerve contributions to the anterolateral abdominal wall arise from T11 and T12. Electrophysiological confirmation of these findings was obtained through intraoperative stimulation. Therefore, we believe that the occurrence of postoperative abdominal flank

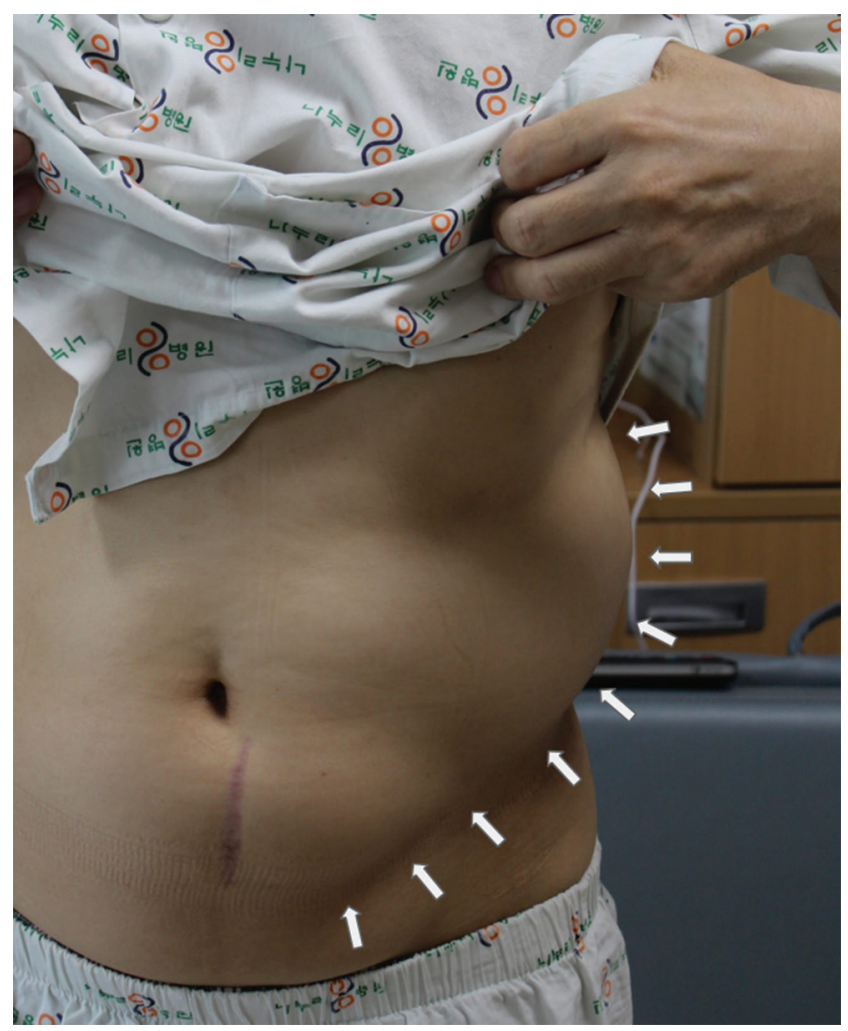

Fig. 5 Photography shows left abdominal flank bulging (white arrow).

bulge is most likely attributable to muscle denervation caused by injury to the T11 and T12 intercostal nerves.

It is possible that postoperative abdominal flank bulging is caused by direct muscular injury and disruption of the local blood supply to the muscle around the incision. However, there is abundant evidence that implicates denervation injury 


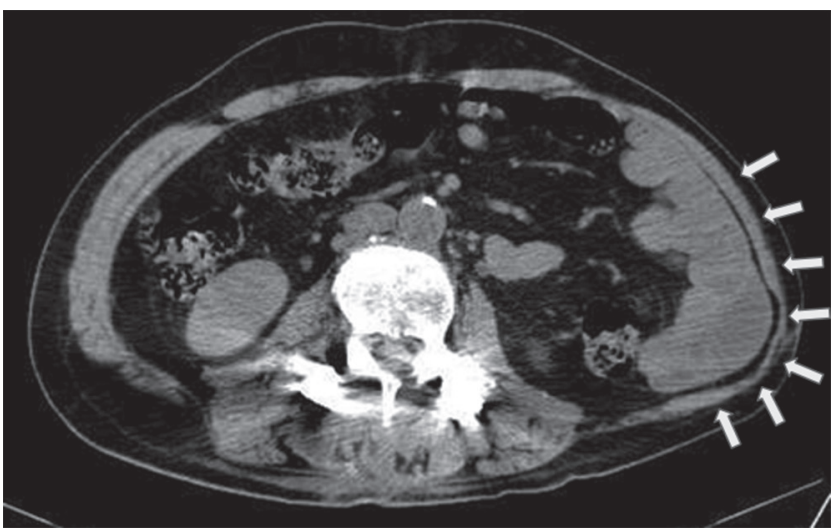

Fig. 6 CT scan shows left abdominal flank bulging accompanied by abdominal muscle thinning, and no evident of abdominal facial defect and bowel hernia (white arrow).

as the more likely etiology. Multiple authors have suggested that abdominal flank bulging after retroperitoneal approaches may be a result of denervation injury. ${ }^{6,8-10)}$ The pattern of muscular atrophy has even been documented as following an oblique pattern, mimicking a dermatomal distribution. ${ }^{11)}$

It is very important to distinguish between a true hernia and a denervation injury of the anterolateral abdominal musculature. True hernias involve one or more facial defects, whereas a denervation injury involves thinning of the musculature with an intact facial plane. ${ }^{12)}$ If it is a true hernia, a reoperation for repair is required. Whereas, if it is a denervation injury, it is only necessary to observe the abdominal flank bulging. In our case, the abdominal flank bulging showed an oblique and dermatomal pattern below the skin incision. An abdominal CT scan showed no facial defect. Therefore, it is not an incisional hernia. It is abdominal flank bulging due to abdominal muscle weakness caused by injury to the T11 intercostal nerve.

One article reported three patients with an abdominal wall paralysis after open thoracic surgery. ${ }^{6}$ In two of three cases, the abdominal wall paralysis resolved within a few months. In the remaining case, the condition was still present 1 year after the operation. The authors of the report concluded that damage to the intercostal nerves could be reduced with less invasive techniques. Another article reported that awareness of the anatomical course of the intercostal nerve is the first step in preventing abdominal flank bulging. ${ }^{8)}$ They recommend protecting the intercostal nerve with moistened cottonoid while coagulating the intercostal artery or vein. They also recommend protecting the intercostal nerve along the rostral rib with a moistened laparotomy sponge when placing the rib spreader.

Abdominal flank bulging is an unsightly and painful complication that can occur after a lateral retroperitoneal approach to the thoracolumbar spine. The most significant intercostal nerve contributing to the anterolateral abdominal wall arises from T11 and T12. Therefore, this complication results from denervation of the abdominal musculature, caused by injury to the T11 and T12 intercostal nerves. To consider the case of this complication for focused on our operating procedure, the causes of this complication are excessive irritation of intercostal neurovascular bundle during periosteal dissection using dissector, or excessively long periods of retraction of the 10th and 12th rib, including the 11th intercostal neurovascular bundle, to secure a wide operational view. We believe that a retraction injury is more likely to develop in the abdominal flank bulge than an irritation injury sustained during periosteal dissection. Awareness of the anatomical course of the intercostal nerve, a meticulous approach intended to minimize intercostal nerve injury, and awareness of rib and intercostal neurovascular bundle retraction is the first step in preventing this complication.

\section{Conflict of Interest}

The authors declare that they have no conflict of interest.

\section{Informed Consent}

Additional informed consent was obtained from all individual participants for whom identifying information is included in this article.

\section{References}

1) Park Y, Ha JW: Comparison of one-level posterior lumbar interbody fusion performed with a minimally invasive approach or a traditional open approach. Spine (Phila Pa 1976) 32: 537-543, 2007

2) Arnold PM, Anderson KK, McGuire RA: The lateral transpsoas approach to the lumbar and thoracic spine: a review. Surg Neurol Int 3: S198-S215, 2012

3) Sofianos DA, Briseño MR, Abrams J, Patel AA: Complications of the lateral transpsoas approach for lumbar interbody arthrodesis: a case series and literature review. Clin Orthop Relat Res 470: 16211632,2012

4) Graham RB, Wong AP, Liu JC: Minimally invasive lateral transpsoas approach to the lumbar spine: pitfalls and complication avoidance. Neurosurg Clin N Am 25: 219-231, 2014

5) Wang MY, Mummaneni PV: Minimally invasive surgery for thoracolumbar spinal deformity: initial clinical experience with clinical and radiographic outcomes. Neurosurg Focus 28: E9, 2010

6) Timmermans L, Klitsie PJ, Maat AP, de Goede B, Kleinrensink GJ, Lange JF: Abdominal wall bulging after thoracic surgery, an underdiagnosed wound complication. Hernia 17: 89-94, 2013

7) Rogers ML, Duffy JP: Surgical aspects of chronic post-thoracotomy pain. Eur J Cardiothorac Surg 18: 711-716, 2000

8) Fahim DK, Kim SD, Cho D, Lee S, Kim DH: Avoiding abdominal flank bulge after anterolateral approaches to the thoracolumbar spine: cadaveric study and electrophysiological investigation. J Neurosurg Spine 15: 532-540, 2011

9) Gardner GP, Josephs LG, Rosca M, Rich J, Woodson J, Menzoian JO: The retroperitoneal incision. An evaluation of postoperative flank 'bulge.' Arch Surg 129: 753-756, 1994

10) Hoffman RS, Smink DS, Noone RB, Noone RB Jr, Smink RD: Surgical repair of the abdominal bulge: correction of a complication of the flank incision for retroperitoneal surgery. J Am Coll Surg 199: 830-835, 2004

11) Goodman $P$, Balachandran S: Postoperative atrophy of abdominal wall musculature: CT demonstration. J Comput Assist Tomogr 15: 989993, 1991

12) Salameh JR, Salloum EJ: Lumbar incisional hernias: diagnostic and management dilemma. JSLS 8: 391-394, 2004 